\title{
Contribution of perfusion-weighted magnetic resonance imaging in the differentiation of meningiomas and other extra-axial tumors: case reports and literature review
}

\author{
Anna Zimny $\cdot$ Marek Sasiadek
}

Received: 18 July 2010/Accepted: 21 October 2010/Published online: 9 November 2010

(C) The Author(s) 2010. This article is published with open access at Springerlink.com

\begin{abstract}
We present six cases of extra-axial lesions: three meningiomas [including one intraventricular and one cerebellopontine angle (CPA) meningioma], one dural metastasis, one CPA schwannoma and one choroid plexus papilloma which were chosen from a larger cohort of extraaxial tumors evaluated in our institution. Apart from conventional MR examinations, all the patients also underwent perfusion-weighted imaging (PWI) using dynamic susceptibility contrast method on a $1.5 \mathrm{~T}$ MR unit (contrast: $0.3 \mathrm{mmol} / \mathrm{kg}$, rate $5 \mathrm{ml} / \mathrm{s}$ ). Though the presented tumors showed very similar appearance on conventional MR images, they differed significantly in perfusion examinations. The article draws special attention to the usefulness of PWI in the differentiation of various extra-axial tumors and its contribution in reaching final correct diagnoses. Finding a dural lesion with low perfusion parameters strongly argues against the diagnosis of meningioma and should raise a suspicion of a dural metastasis. In cases of CPA tumors, a lesion with low relative cerebral blood volume values should be suspected to be schwannoma, allowing exclusion of meningioma to be made. In intraventricular tumors arising from choroid plexus, low perfusion parameters can exclude a diagnosis of meningioma. In our opinion, PWI as an easy and quick to perform functional technique should be incorporated into the MR protocol of all intracranial tumors including extra-axial neoplasms.
\end{abstract}

Keywords Magnetic resonance imaging . Brain perfusion $\cdot$ Extra-axial tumors $\cdot$ Meningiomas

\footnotetext{
A. Zimny $(\bowtie) \cdot$ M. Sasiadek

Department of General and Interventional Radiology and

Neuroradiology, Wroclaw Medical University,

Borowska Street 213, 50-556 Wroclaw, Poland

e-mail: abernac@wp.pl
}

\section{Introduction}

Intracranial extra-axial neoplasms are tumors arising from tissues other than brain parenchyma, such as meninges, dura, calvarium, ventricles, choroid plexus, pineal gland or pituitary gland, with meningiomas being the most common type comprising 13-26\% of all intracranial neoplasms in adults [1]. Meningiomas originate from arachnoid meningothelial cells and appear as broad-based dural hemispheric or oval lesions, attached to the dura mater. They most frequently occur supratentorially at calvaria or skull base meninges, along falx and in the parafalcine location, but they can also be found attached to tentorium, in the cerebellopontine angle (CPA), within the optic nerve or intraventricularly. Though they have a fairly characteristic appearance on CT and MRI, they need to be differentiated from other extra-axial lesions that can strongly mimic meningiomas both clinically and radiologically, such as metastases, primary glial and mesenchymal tumors, hematopoetic neoplasms (lymphomas, plasmocytomas) or even inflammatory (reumathoid arthritis) and infectious diseases (tuberculosis) [2]. If located in the CPA, meningiomas can be difficult to distinguish from vestibular schwannomas, and if located within ventricles, they need to be differentiated from other ventricle tumors such as choroid plexus papillomas or ependymomas.

As many extra-axial lesions are indistinguishable from meningiomas using conventional MRI, the advanced MR techniques such as MR spectroscopy (MRS) and perfusionweighted imaging (PWI) can be applied to obtain more information on tumor biology. MRS has been widely used in glial tumors but its application in extra-axial tumors is still limited. MRS is capable of the differentiation of various extra-axial tumors on the basis of metabolite spectra (high alanine and low $N$-acetylaspartate peaks in meningiomas, 
high myoinositol in schwannomas, and presence of lactate/ lipid peaks in metastases) [3]. On the other hand, MRS can be difficult to perform in extra-axial tumors due to their location near the skull and bone artefacts especially in the CPA, thus making this technique limited mainly to large lesions [4].

PWI is a method that provides information on cerebral physiology at the capillary level (microvasculature). Among a few PWI techniques, dynamic susceptibility contrast (DSC) MR imaging is the most often used. The method is based on the measurements of the MR signal using T2*weighted sequence during the first pass of a bolus of a paramagnetic contrast agent [5]. DSC MRI provides maps of cerebral blood volume (CBV) and noninvasive measurements of relative cerebral blood volume (rCBV). In brain tumors, $\mathrm{rCBV}$ is defined as the ratio between $\mathrm{CBV}$ in the tumor and $\mathrm{CBV}$ in the white matter of the contralateral hemisphere. rCBV parameter correlates with tumor vascularity and is increased in tumors with a high rate of pathologic neoangiogenesis [6]. In glial tumors, increased rCBV ratios indicate increased malignancy, but this rule cannot be applied to extra-axial tumors - there are highly vascular extra-axial tumors, e.g., meningiomas, which are benign in terms of biological behavior [7].

Since most of the PWI studies of intracranial tumors have focused on glial neoplasms, this article draws special attention to the usefulness of PWI in differentiation of various extra-axial tumors and its contribution in reaching a final correct diagnosis. We present six cases of extraaxial tumors which show very similar appearance on conventional MR images and differ significantly in perfusion examination.

\section{Materials and methods}

We present six cases of extra-axial lesions: three meningiomas (including one intraventricular and one CPA meningioma), one dural metastasis, one CPA schwannoma and one choroid plexus papilloma. All patients underwent conventional MR imaging followed by DSC MRI. The cases were selected from a cohort of 32 extra-axial tumors evaluated with PWI in our institution in the years 2008-2009. The histopathological diagnosis was established in all patients by a neuropathologist according to the World Health Organization classification system for tumors of the central nervous system.

\section{Data acquisition}

The imaging examinations were performed with $1.5 \mathrm{~T}$ MR unit (Signa Hdx; GE Medical Systems) using a 16-channel coil dedicated for head and spine imaging. Before contrast injection, standard brain protocol was applied: non-enhanced axial T1-weighted images, axial, coronal and sagittal T2-weighted images and axial fluid attenuated inversion recovery sequences were obtained. Then, DSC MR imaging was performed using gradient echo planar T2*-weighted sequence with the following parameters: $\mathrm{TR}=1.900 \mathrm{~ms}, \mathrm{TE}=80 \mathrm{~ms}, \mathrm{FOV}=30 \mathrm{~cm}$, matrix $=$ $192 \times 128$, slice thickness $=8 \mathrm{~mm}$ without spacing, NEX 1.0. Ten seconds after the start of the image acquisition, a bolus of a 1.0-mmol/1 gadobutrol formula (Gadovist; Schering Bayer Pharma) in a dose of $0.3 \mathrm{mmol} / \mathrm{kg}$ of body weight (as indicated by the producing company) was injected via 20-gauge catheter placed in the antecubital vein. Contrast administration was performed using an automatic injector (Medrad) at a rate of $5 \mathrm{ml} / \mathrm{s}$ and was followed by a saline bolus $(20 \mathrm{ml}$ at $5 \mathrm{ml} / \mathrm{s})$. The whole perfusion imaging lasted $1 \mathrm{~min} 26 \mathrm{~s}$, in which sets of images from 13 axial slices parallel to the anterior commisure-posterior commisure plane were obtained before, during and after contrast injection. After dynamic studies, postcontrast T1-weighted 3D images were performed using contrast administered for perfusion examination.

\section{Image postprocessing}

The dynamic images were postprocessed using Functool software (ADW 4.4; GE Medical Systems). CBV maps were computed on a pixel-wise basis from the first-pass data as described by Rosen et al. [5]. CBV values obtained with this methodology are not absolute measurements and require to be related to the reference standard. All CBV values obtained within the tumor core were normalized to the CBV values from the normal-appearing contralateral white matter. In each tumor, color-coded perfusion maps as well as rCBV values and time-intensity curves were analyzed.

\section{Results}

Case 1 (Fig. 1a-c)

A 50-year-old male. MR images demonstrated a dural tumor located along falx with broad attachment to meninges. The lesion was hypointense on T1- and hyperintense on T2-weighted images with mild surrounding edema. After contrast injection, the tumor enhanced strongly, mostly homogeneously, with central necrosis; peritumoral dural enhancement (dural tail sign) was also visible. Moderate mass effect was present. Perfusion maps showed marked hyperperfusion $(\mathrm{rCBV}=18.7)$, and the time-intensity curve revealed strong signal drop with no return to the baseline. Histopathologically angiomatous meningioma was diagnosed. 

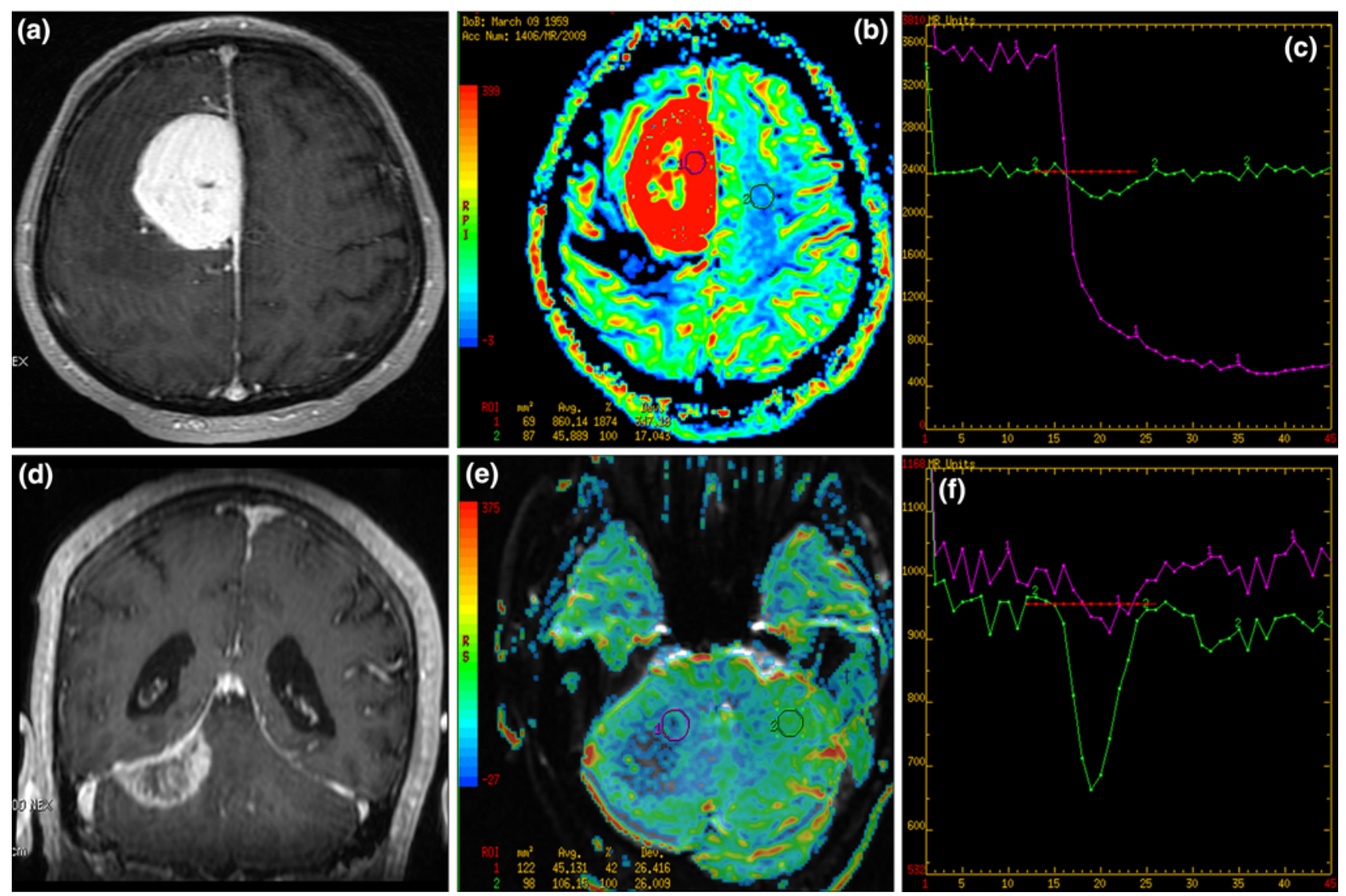

Fig. 1 Two dural lesions: T1 post-contrast (a, d) images, CBV maps $(\mathbf{b}, \mathbf{e})$ and time-intensity curves $(\mathbf{c}, \mathbf{f})$. a-c Parafalcine meningioma with high rCBV values (red coloring) on perfusion maps and the typical time-intensity curve with no return to the baseline after high

\section{Case 2 (Fig. 1d-f)}

A 58-year-old male. MR images demonstrated a meningioma-like dural tumor located along tentorium on the right side in the posterior cranial fossa. The lesion showed signal intensity similar to cerebellar parenchyma on T1-weighted images, while on T2-weighted images the tumor was hypointense. Mild peritumoral edema was also seen. After contrast injection, heterogeneous enhancement and the dural tail sign were noticed. No other enhancing pathological lesions were found within the brain. Perfusion maps showed very low $\mathrm{rCBV}$ values $(\mathrm{rCBV}=0.4)$ with the time-intensity curve returning to the baseline. The patient did not have any history of a neoplastic disease. Gross pathology revealed dural metastasis of the squamous cell carcinoma of the lungs.

\section{Case 3 (Fig. 2a-c)}

A 54-year-old male. MR images showed a CPA tumor arising from the right internal auditory canal (IAC) which was moderately enlarged. The tumor was hypointense on T1- and hyperintense on T2-weighted images and showed signal drop (violet curve). d-f Dural metastasis mimicking meningioma on conventional MR images but with low rCBV values (blue coloring) and the time-intensity curve returning to the baseline (violet curve)

strong homogeneous enhancement after contrast administration. Moderate mass effect was noticed. rCBV map showed hypoperfusion $(\mathrm{rCBV}=0.5)$ and the time-intensity curve returned to the baseline. Histopathological evaluation revealed schwannoma.

\section{Case 4 (Fig. 2d-f)}

A 51-year-old male. MR images demonstrated a CPA tumor with attachment to meninges and an intracanalicular part detected after contrast administration. The lesion was isointense on T1- and hypointense on T2-weighted images and showed strong homogeneous enhancement after contrast injection. Perfusion maps revealed high $\mathrm{rCBV}$ values $(\mathrm{rCBV}=7.0)$, and the time-intensity curve a pronounced signal drop with no return to the baseline. Histopathology revealed fibrous meningioma.

Case 5 (Fig. 3a-c)

A 23-year-old male. MR images demonstrated intraventricular tumor located in the body of the left lateral 

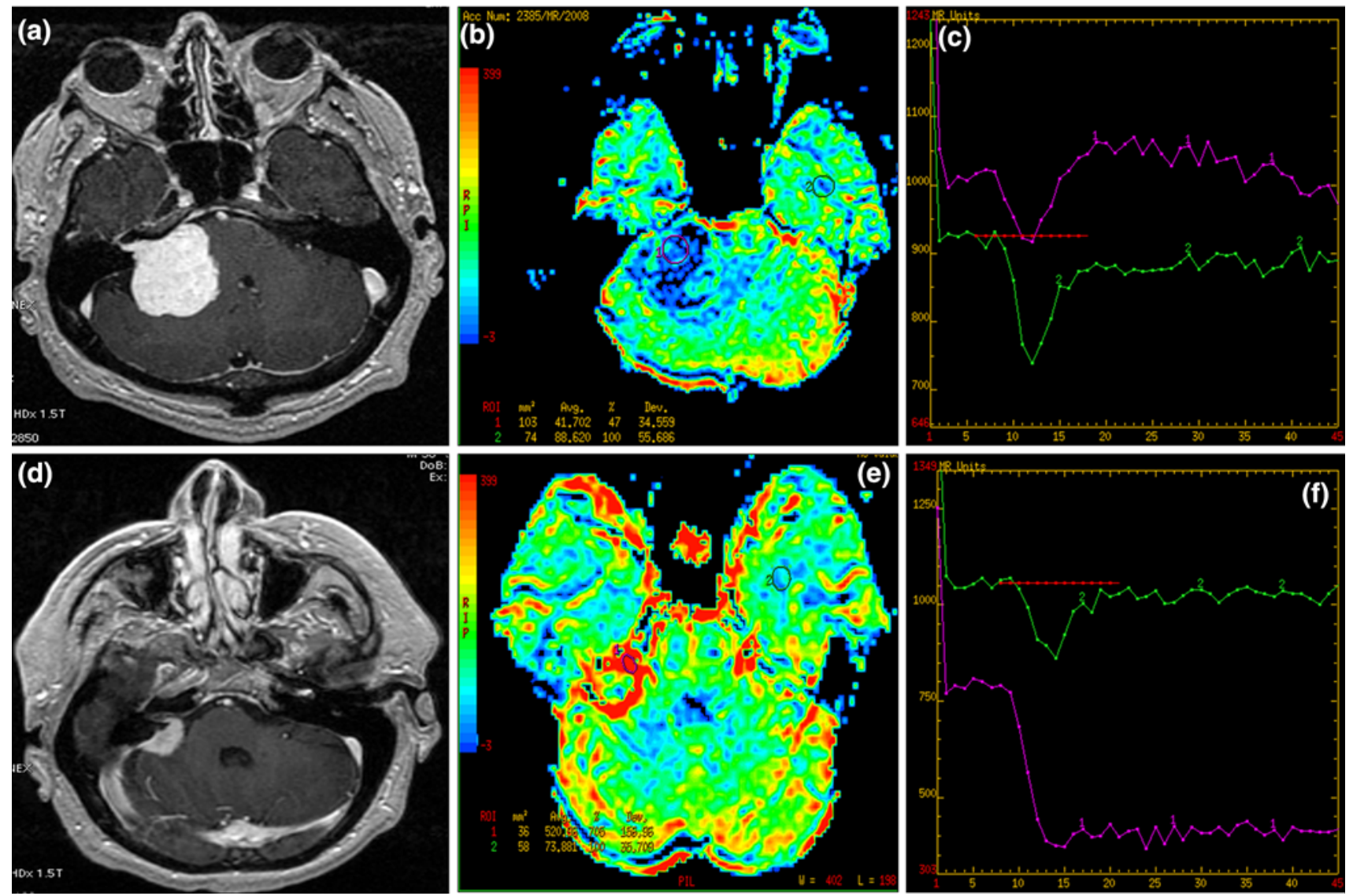

Fig. 2 Two CPA tumors: T1 post-contrast (a, d) images, CBV maps $(\mathbf{b}, \mathbf{e})$ and time-intensity curves $(\mathbf{c}, \mathbf{f})$. a-c Typical schwannoma with low perfusion parameters (blue coloring) and the time-intensity curve returning to the baseline (violet curve). d-f Meningioma with

ventricle. On T1- and T2-weighted images, the lesion was isointense to the cortex and enhanced strongly, but slightly heterogeneously, after contrast administration. Mild ventricle enlargement was seen. Perfusion maps showed hypoperfusion in the center $(\mathrm{rCBV}=0.65)$ with moderately elevated rCBV values (up to 1.8 ) peripherally. Perfusion time-intensity curve did not return to the baseline. Histopathological evaluation revealed a choroid plexus papilloma.

\section{Case 6 (Fig. 3d-f)}

A 23-year-old female. MR images showed intraventricular tumor located in the trigone of the left lateral ventricle. On T1- and T2-weighted images, the lesion was isointense to the cortex and enhanced strongly and homogeneously after contrast injection. Perfusion maps showed pronounced hyperperfusion $(\mathrm{rCBV}=10.4)$ and the time-intensity curve demonstrated no return to the baseline after strong signal drop. Histopathology revealed intraventricular meningioma. intracanalicular enhancing part showing typical hyperperfusion (red coloring) and the time-intensity curve with no return to the baseline (violet curve)

\section{Discussion}

Meningiomas are the most common primary nonglial neoplasms with typical radiological features. On CT, they usually appear as hyperdense, often calcified lesions; on MRI, they are mostly isointense with cortex on all sequences. Meningiomas enhance strongly after contrast injection and often homogeneously, but areas of necrosis especially in large lesions can also be seen. Though not specific for meningiomas, they very often produce the socalled dural tail sign which is enhancing thickened peritumoral dura [8].

Meningiomas are highly vascular tumors, and in PWI, they appear as hyperperfused lesions with elevated $\mathrm{rCBV}$ values. In several DSC examinations, mean $\mathrm{rCBV}$ values for all meningiomas were reported to range between 6 and $9[9$, $10]$ and were lower for meningothelial, fibrous and anaplastic meningiomas (mean $\mathrm{rCBV}=5-7$ ) and higher for angiomatous meningiomas (mean $\mathrm{rCBV}=11.9$ ) [11]. Also, in all our cases of meningiomas, high perfusion results were demonstrated $(\mathrm{rCBV}=7.05$ for $\mathrm{CPA}$ meningioma, $\mathrm{rCBV}=10.4$ for intraventricular meningioma) with the 

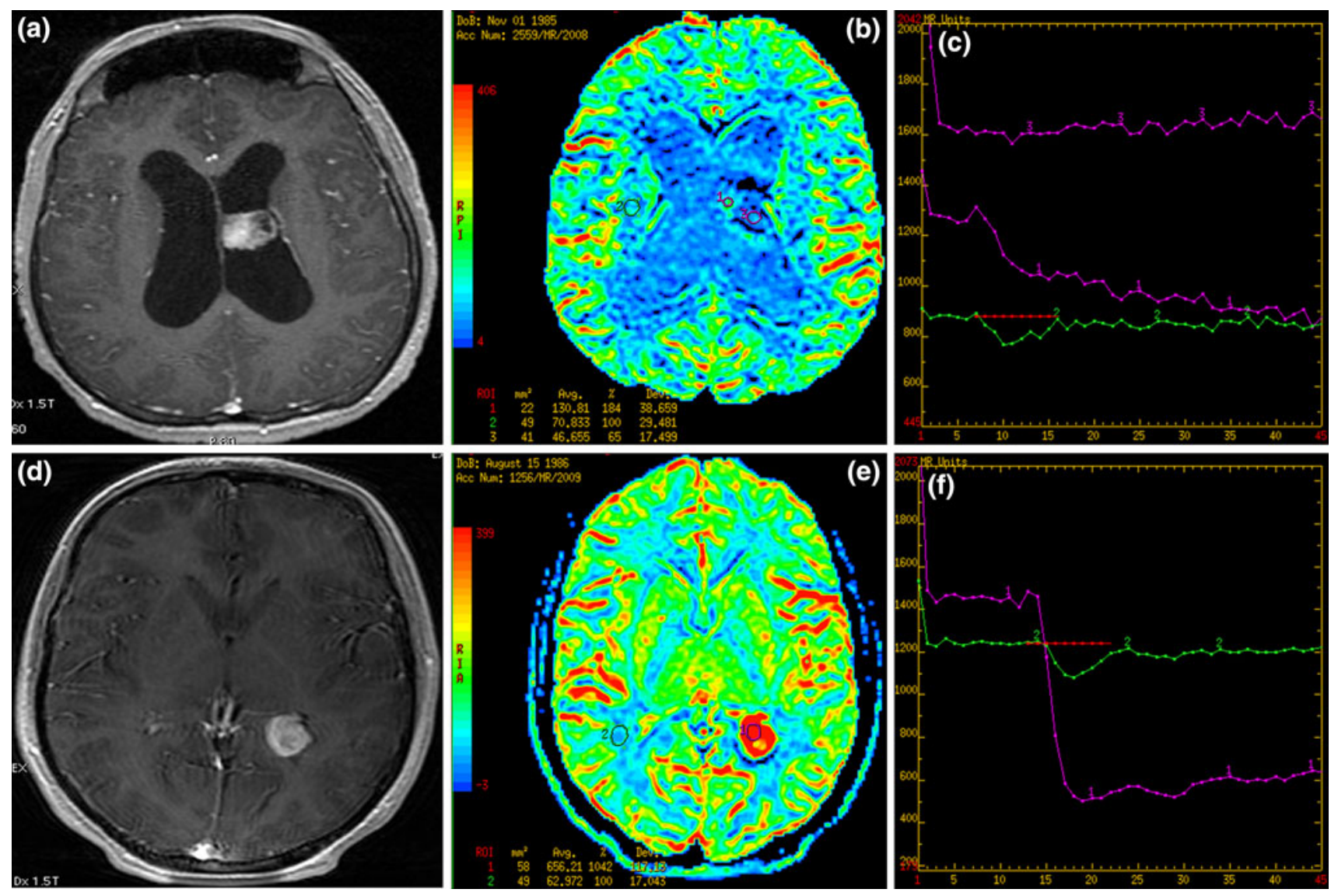

Fig. 3 Two intraventricular tumors: T1 post-contrast (a, d) images, CBV maps $(\mathbf{b}, \mathbf{e})$ and time-intensity curves $(\mathbf{c}, \mathbf{f})$. a-c choroid plexus papilloma with low rCBV values on perfusion maps (blue and green coloring) and the time-intensity curves showing no return to the

most elevated rCBV values found in angiomatous meningioma $(\mathrm{rCBV}=18.7)$.

Apart from characteristic rCBV values, meningiomas usually show typical time-intensity curves with minimal or no return to the baseline level [12]. This phenomenon is due to the absence of the blood-brain barrier in neovessels of these tumors and thus the high rate of extracapillary leakage of contrast material. The typical time-intensity curves were seen in all our cases of meningiomas regardless of their location within falx, in the CPA or intraventricularly (Figs. 1c, 2f and 3f).

Though meningiomas are the most common dural tumors, they need to be differentiated with other meningeal lesions, in particular meningeal metastases, from prostate, lung, kidney or breast cancers [13]. If lesions arise multifocally within meninges, they are highly suggestive of metastases, but if they present as a solitary tumor especially in a patient without previous history of primary malignancy like in our case 2 , they can cause severe diagnostic problems. Isolated dural metastases representing less than $1 \%$ of intracranial metastases [14] strongly mimic meningiomas, baseline (violet curves). $\mathbf{d}-\mathbf{f}$ intraventricular meningioma with typical high rCBV values (red coloring) and the time-intensity curve with no return to the baseline (violet curve)

and less than 40 cases of dural metastases interpreted as meningiomas have been previously reported [2, 4, 13, 15]. Kremer et al., evaluating 6 dural metastases and 16 meningiomas, did not find any significant differences between those lesions on conventional MR images [10]. Both dural metastases and meningiomas showed similar appearance on T1- and T2-weighted images, similar heteroor homogeneous contrast enhancement pattern, and a dural tail sign. Also, in our cases 1 and 2, plain MR images were not sufficient to discriminate both lesions. Though the two lesions looked very similar on conventional MR images, we noticed significant differences between them in PWI-a meningioma showed typical very high (18.7) and a metastasis low (0.4) CBV ratios. Our findings are consistent with the previous reports from perfusion studies of meningiomas and dural metastases. Kremer et al. found that the mean rCBV values of 16 meningiomas (mean $\mathrm{rCBV}=8.97$, range 4-18) were significantly higher than those of dural metastases of a lung carcinoma (one case: 1.26), lymphoma (one case: 1.29), breast carcinoma (two cases: $1.5,1.56$ ) and rectal carcinoma (one case: 3.34) [10]. It also has to be 
stressed that the majority of dural metastases show low perfusion results, but not all of them. Hypervascular lesions such as Merkel carcinoma, kidney carcinoma or melanoma metastases may present elevated $\mathrm{rCBV}$ values which make them indistinguishable from meningiomas [10, 16].

High-grade astrocytomas invading the dura mater may also be difficult to distinguish from meningiomas on plain MRI. Moreover, both lesions show high rCBV values in PWI $[6,9]$. Cha et al. suggest that in those cases evaluation of the time-intensity curve during the first pass is of special importance and can be helpful in differentiating between these two tumor types. In extra-axial tumors, the curve does not return to baseline, which was also seen in all our cases of meningiomas (Figs. 1c, $2 \mathrm{f}$ and $3 \mathrm{f}$ ), whereas in high-grade gliomas, there is a significant partial return of the curve to the baseline levels [12]. Other dural lesions mimicking meningiomas such as infectious or inflammatory diseases are rare and will not be discussed [2].

Meningiomas located in the CPA account for 10-15\% of all lesions in this location and should in particular be differentiated from vestibular schwannomas accounting for $70-80 \%$ of all CPA lesions $[17,18]$. Other CPA lesions are rare, with the epidermoid cyst being the most frequent (5\%) [17]. Most vestibular schwannomas develop from the Schwann cells in the sheath of the inferior vestibular nerve in the IAC. When they grow bigger and give rise to a round or oval component in the CPA cistern, schwannomas can be typically seen as "ice on cone" lesions. On MR images, they show T1-isointensity and T2 high signal intensity, homo- or heterogeneous enhancement and may contain cystic or necrotic components, especially in lesions exceeding $2.5 \mathrm{~cm}$ [19]. The differential diagnostic problems can be caused by larger schwannomas without an obvious intracanalicular part and with a dural tail (suggestive of meningioma) or by meningiomas with dural enhancement within IAC (suggestive of schwannoma) like in our case 4. Because MR image is not always conclusive, PWI may be helpful to solve difficult cases. The rCBV values evaluated with DSC MRI are reported to be significantly lower in schwannomas than in meningiomas [9, 20], with the mean rCBV values 3.23 and 8.02, respectively. Even if there is an overlap between $\mathrm{rCBV}$ ratios of both entities, the highest reported ratios in schwannomas did not exceed 4.4 while the mean rCBV ratio of typical meningiomas ranges from 6 to 9 [7, 9, 10]. In our cases 3 and 4 , perfusion results were similar to those reported in the literature. Though both tumors enhanced strongly after contrast administration, they showed different perfusion results-low rCBV values in schwannoma (Fig. 2b) and high rCBV values in meningioma (Fig. 2e). It is important to remember that the pattern of perfusion within the tumor does not reproduce enhancement characteristics found in the post-contrast MRI, since in conventional MRI contrast enhancement represents areas of disrupted blood-brain barrier rather than tumor vascularity per se [21, 22]. Areas of hyperperfusion in PWI can be identified in both enhancing and nonenhancing tumor regions, and conversely strongly enhancing tumors may show both high or low perfusion results in PWI. This is the case in schwannomas and meningiomas which both strongly enhance in conventional post-contrast MRI studies due to the lack of the blood-brain barrier and significant permeability of tumor vessels for the contrast agent. Their different perfusion pattern reflects the rate of tumor vascularity which is low in schwannomas and high in meningiomas [20].

Our cases 5 and 6 raise a problem of differentiation of intraventricular lesions. Intraventricular tumors represent a diverse group of lesions, some of them infrequent. The most useful indication for the diagnosis are patients' age and precise intraventricular location. The most common tumors arising from the choroid plexus are choroid plexus papillomas, choroid plexus carcinomas, meningiomas and metastases [23].

Choroid plexus papillomas account for $0.4-0.6 \%$ of all intracranial tumors and nearly half of these tumors manifest in the first decade of life [24]. They usually arise in the lateral or the forth ventricle. Choroid plexus papillomas are cauliflower-like masses with prominent lobulations peripherally, calcifications, hemorrhage and cyst formation seen occasionally. They appear as iso- or hyperdense masses on CT and iso- or hypointense structures on T1weighted and heterogeneous on T2-weighted images. They show strong enhancement on both CT and MR images. Hydrocephalus is very common due to an increase in the CSF production by the tumor [23, 24]. There are no reports in the literature on the results of spectroscopic or perfusion examinations in those tumors. Our study demonstrates low values of rCBV in choroid plexus papilloma (Fig. 3b).

Intraventricular meningioma is a rare tumor, constituting approximately $0.5-2.0 \%$ of all intracranial meningiomas, but this tumor is still one of the most common intraventricular neoplasms in the adult population [24, 25]. The majority of them occur after the fourth decade of life though they might be present earlier like in our case 6 . Intraventricular meningiomas mostly occupy trigone of the lateral ventricle and are believed to arise from the arachnoidal cap cells trapped within the choroid plexus [24]. Their appearance on conventional imaging studies is similar to that of other meningiomas [24, 25]. MRS of intraventricular meningiomas has also revealed a pattern similar to that of meningiomas outside the ventricular system with decreased amounts of NAA, and increased amounts of lactate, lipids and alanine [25]. To our knowledge, there are no reports on the perfusion appearance of intraventricular meningiomas. Our study proves that the perfusion results in intraventricular meningiomas resemble those of other 
meningiomas with high $\mathrm{rCBV}$ values and the time-intensity curve showing no return to the baseline (Fig. 3e, f).

Cases 5 and 6 show that choroid plexus papilloma and intraventricular meningioma, though appearing similar on conventional MR images, demonstrate different patterns of perfusion: low rCBV values and high rCBV values, respectively. We believe that DSC MRI can be helpful in distinguishing those intraventricular lesions, though further investigations including more cases are required to find the common perfusion patterns especially in choroid plexus papillomas.

\section{Conclusions}

Optimal management of extra-axial lesions requires an accurate preoperative diagnosis and PWI can provide additional information helpful in distinguishing dural metastases from meningiomas, CPA meningiomas from schwannomas or intraventricular meningiomas from choroid plexus papillomas.

Finding a dural lesion with low perfusion parameters strongly argues against diagnosis of a meningioma and should raise a suspicion of a dural metastasis. In cases of CPA tumors, a lesion with low rCBV values should be suspected to be schwannoma, allowing the exclusion of a meningioma to be made. In intraventricular tumors arising from choroid plexus, low perfusion parameters can exclude a diagnosis of a meningioma.

In our opinion, PWI as an easy and quick to perform functional technique should be incorporated into the MR protocol of all intracranial tumors including extra-axial neoplasms.

Open Access This article is distributed under the terms of the Creative Commons Attribution Noncommercial License which permits any noncommercial use, distribution, and reproduction in any medium, provided the original author(s) and source are credited.

\section{References}

1. Engelhard HH (2001) Progress in the diagnosis and treatment of patients with meningiomas: diagnostic imaging, preoperative embolization. Surg Neurol 55:89-101

2. Johnson MD, Powell S, Boyer P et al (2002) Dural lesions mimicking meningiomas. Human Pathol 33:1211-1226

3. Cho YD, Choi GH, Lee SP et al (2003) $\mathrm{H}^{1}$-MRS metabolic patterns for distinguishing between meningiomas and other brain tumors. Magn Reson Imaging 21:663-672

4. Scherer K, Johnston J, Panda M (2009) Dural based mass: malignant or benign. Radiol Case 3(11):1-12
5. Rosen B, Belliveau J, Vevea J et al (1990) Perfusion imaging with NMR contrast agents. Magn Reson Med 14:249-265

6. Aronen HJ, Gazit IF, Louis DN (1994) Cerebral blood volume maps of gliomas: comparison with tumor grade and histologic findings. Radiology 191:41-51

7. Yang S, Law M, Zagzag D (2003) Dynamic contrast-enhanced perfusion MR imaging measurements of endothelial permeability: differentiation between atypical and typical meningiomas. Am J Neuroradiol 24:1554-1559

8. Guermazi A, Lafitte F, Miaux Y et al (2005) The dural tail signbeyond meningioma. Clin Radiol 60:171-188

9. Hakyemez B, Erdogan C, Bolca N et al (2006) Evaluation of different cerebral mass lesions by perfusion-weighted MR imaging. J Magn Reson Imaging 24:817-824

10. Kremer S, Grand S, Remy C et al (2004) Contribution of dynamic contrast MR imaging to the differentiation between dural metastasis and meningioma. Neuroradiology 46:642-648

11. Zhang H, Rodiger LA, Shen T, Miao J, Oudkerk M (2008) Preoperative subtyping of meningiomas by perfusion MR imaging. Neuroradiology 50:835-840

12. Cha S, Knopp EA, Johnson G et al (2002) Intracranial mass lesions: dynamic contrast-enhanced susceptibility-weighted echoplanar perfusion MR imaging. Radiology 223:11-29

13. Tagle P, Villanueva $P$, Torrealba $G$ et al (2002) Intracranial metastasis or meningioma? An uncommon clinical diagnostic dilemma. Surg Neurol 58:241-245

14. Simionescu M (1960) Metastatic tumors of the brain: a follow-up study of 195 patients with neurosurgical considerations. J Neurosurg 17:361-373

15. Rumana C, Hess K, Shi W et al (1998) Metastatic brain tumors with dural extension. J Neurosurg 89:552-558

16. Kremer S, Grand S, Berger F et al (2003) Dynamic contrastenhanced MRI: differentiating melanoma and renal carcinoma metastases from high-grade astrocytomas and other metastases. Neuroradiology 45:44-49

17. Bonneville F, Savatovsky J, Chiras J (2007) Imaging of cerebellopontine angle lesions: an update. Part 1: enhancing extraaxial lesions. Eur Radiol 17:2472-2482

18. Sarrazin JL (2006) Infratentorial tumors. J Radiol 87:748-763

19. Duvoisin B, Fernandes J, Doyon D et al (1991) Magnetic resonance findings in 92 acoustic neuromas. Eur J Radiol 13:96-102

20. Maeda M, Itoh S, Kimura H et al (1994) Vascularity of meningiomas and neuromas: assessment with dynamic susceptibilitycontrast MR imaging. Am J Roentgenol 163:181-186

21. Grossman I, Wolf G, Biery D et al (1984) Gadolinium-enhanced nuclear magnetic resonance images of experimental brain abscess. J Comput Assist Tomogr 8:204-207

22. Brasch RC, Weinmann HJ, Wesbey GE (1984) Contrastenhanced NMR imaging: animal studies using gadolinium-DTPA complex. Am J Roentgenol 142:625-630

23. Guermazi A, de Kerviler E, Zagdanski AM, Frija J (2000) Diagnostic imaging of choroid plexus disease. Clin Radiol 55: 503-516

24. Koeller KK, Sandberg GD (2002) Cerebral intraventricular neoplasms: radiologic-pathologic correlation. RadioGraphics 22: 1473-1505

25. Majos C, Cucurella G, Aguilera C, Coll S, Pons LC (1999) Intraventricular meningiomas: $\mathrm{MR}$ imaging and MR spectroscopic findings in two cases. Am J Neuroradiol 20:882-885 